\title{
Value of Repeated Direct Smear Sputum Examination in the Diagnosis of Pulmonary Tuberculosis
}

\author{
Wafaa Ali Hassan1*, Amany Omar1, Saher Khalil'2, Atef Farouk Al-Qarn1 \\ ${ }^{1}$ Chest Department, Assiut University, Assiut, Egypt \\ ${ }^{2}$ Qena Chest Hospital, Qena, Egypt \\ Email: wafaagadallah@yahoo.com
}

Received 16 March 2014; revised 31 March 2014; accepted 8 April 2014

Copyright (C) 2014 by authors and Scientific Research Publishing Inc.

This work is licensed under the Creative Commons Attribution International License (CC BY). http://creativecommons.org/licenses/by/4.0/

(c) (i) Open Access

\begin{abstract}
Introduction: The examination of three sputum samples per suspect has been severely criticized from a public health viewpoint and several recent trials have documented the relative inefficiency of the third smear and the necessity for confirmation of a positive smear has also been contested. Aim: This study, undertaken in Qena, Egypt, aimed to determine the usefulness of examining the second and third direct smear microscopy (DSM) specimen in the diagnosis of pulmonary TB. Patients and methods: A retrospective study using record review at TB outpatient clinic; Qena Chest Hospital, Egypt, was done from 2010-2013. Direct smear results were collected as one of the following combinations PNN, PPP, PPN, PNP, NNP, NPP, and NPN, NNN, where $N$ is a negative and $P$ a positive smear. The proportion of positive, first, second and third specimen were calculated. Cases were considered positive having at least one positive smear confirmed by another positive one in the absence of sputum culture. Results: Out of 9420 recorded suspects, 719 of them were positive, so smear positivity was $7.6 \%$. The majority of them were diagnosed from the first sample $(\mathbf{9 6 . 4 \% )}$. For only $3.6 \%$ ( 26 of 719 ), the second smear was positive and a third specimen was required (NPP) to make a definitive diagnosis of TB. No recorded isolated positive or negative smears in the third sample (NNP or PPN). Conclusions: These data indicated that, in our locality with limited financial resources, the incremental yield of a second sputum direct smear examination was low, and the third one was negligible indicating that examination of two sputum samples is enough among pulmonary TB patients. A third sample is required only as confirmatory if the second sample was positive. Smear microscopy can be substantially simplified with favourable resource implications.
\end{abstract}

\section{Keywords}

\section{TB, Direct Smear, Value}

\footnotetext{
${ }^{*}$ Corresponding author.
} 


\section{Introduction}

The resurgence of TB that occurred in the late 1980s and early 1990s resulted in a renewal of interest in improving the efficiency with which laboratories detect M. tuberculosis in clinical specimens [1]. Much of this effort has been directed toward optimizing the methodologies used for the detection of mycobacteria by smear [2], culture [3]-[5], or nucleic acid amplification [6]. Comparatively little attention, however, has been paid to the contribution that specimen number and quality have on the efficiency of the laboratory diagnosis of TB. Smear microscopy with Zeihl-Neelsen (ZN) staining is currently the most feasible microbiological method for diagnosis of pulmonary TB in developing countries due to its rapidity, low cost, and high positive predictive value for Mycobacterium tuberculosis. Most standard laboratory texts [7] and guidelines for mycobacteriology laboratories [8] [9] recommend that at least three sputum specimens, preferably collected on successive days, be submitted to the laboratory for AFB smear and culture for patients suspected to have TB. Unfortunately, there has been a paucity of published data analyzing the validity of this recommendation. It has been recommended that a minimum of 100 high-power fields of microscope should be examined for maximum yield. A minimum of 10 acid fast bacilli (AFB) per 100 fields is taken as the threshold for considering a result as positive, and a definite case should have at least one such result confirmed by a second smear examination or by a suggestive chest radiograph, or alternatively there should be one positive mycobacterial culture result [7] [10]. Some of these guidelines are based on older studies, while for others the arguments are unclear. In particular the examination of three sputum samples per suspect has been severely criticized from a public health viewpoint and several recent trials have documented the relative inefficiency of the third smear [11] [12] while the necessity for confirmation of a positive smear has also been contested [13] .

This study, undertaken in a Governorate of Qena, Egypt, aimed to assess the incremental yield of a second or third sputum sample in pulmonary TB following the existing Revised National Tuberculosis Control Programme guidelines, providing the detailed evaluation needed to determine whether a change in policy is justified.

\section{Patients and Methods}

\subsection{Design}

A retrospective cross-sectional study using record review.

\subsection{Setting}

In Egypt, sputum smear examination forms the cornerstone of both diagnosis as well as monitoring response to treatment. Under the Revised National Tuberculosis Control Programme (RNTCP) all TB patients during the course of their treatment are expected to undergo follow-up sputum smear examination for acid-fast bacilli (AFB) by Ziehl-Neelsen staining at least three times 2Fjournal.pone.0051038" [14]. Sputum smear microscopy was carried out at TB outpatient clinic, Qena chest hospital, Egypt. Suspects of PTB with fever and cough for more than three weeks or symptoms such as anorexia, weight loss and fatigue were asked to submit three sputum specimens for direct smear microscopy over 3 days. Laboratory technicians checked the quantity and quality of specimen that it contained solid or purulent material and not just saliva. Patients were asked to provide a new specimen if only saliva was obtained or volume of less than $3 \mathrm{ml}$ is produced, however if patient failed to provide appropriate sample on repeated visits, then saliva and less volume was processed [15]. Smears were prepared, stained by the Ziehl-Neelsen method according to WHO/IUATLD guidelines and examined. 1\% basic fuchsin and a $0.3 \%$ methylene blue concentrations were used [14]. A positive and negative control slide was included with each run of staining procedure to verify the correct performance of procedure as well as the staining intensity of the acid-fast bacilli. At least 100 microscopic fields were examined to declare a slide negative. In case of positive slides examination even less number of fields are sufficient and the number of organisms present were classified using WHO/IUATLD guidelines [14]. Experienced microbiologist rechecks the positive and all three negative smears. Results were collected as one of the eight possible combinations of smear results as NNN, PNN, PPP, PPN, NNP, NPP, NPN, PNP where N is a negative and P a positive smear. Patients with less than three specimens were registered as incomplete irrespective of the reason and were excluded from final analysis of the incremental yield. Results were aggregated, the proportion of positive, first, second and third specimen were calculated. 


\section{Results}

A total of 10110 suspects for AFB smear microscopy, during a 4-year study period (2010-2013) were recorded (Table 1). $55.5 \%$ of the study subjects were males, and the mean age was $43.26 \pm 17.17$ (Figure 1). Distribution of smears from different cities at Qena governorate and the cause of referral to TB laboratory are shown in Table 2 and Table 3. Of these suspects, 6.8\% (690) gave only two sputum samples and 93.2\% (9420) gave all three sputum samples (Table 4). The results of a total of 9420 AFB examined suspects who submitted three sputum specimens only were included for final analysis. Out of these 9420 suspects, 719 of them were positive (7.6\%), fulfilling the case definition, having at least one positive result confirmed by second smear examination (PPP, NPP). Table 5 shows the results in eight different combinations and Table 6 shows incremental yield of positive identified on first, second and third specimen of 9420 suspects. The percentage of smear positive patients detected annually ranged from $6.4 \%$ to $10.3 \%$. A total of 721 cases had at least one positive smear, $96.2 \%$ (694 of 721) of these first specimens being positive. An isolated negative or positive third sample was not recorded (PPN or NNP), whereas only two cases were positive in either the first or second one (PNN and NPN).

Table 1. Year distribution of the total recorded suspects $(10,110)$.

\begin{tabular}{ccc}
\hline Year & No. & $\%$ \\
\hline $\mathbf{2 0 1 0}$ & 2939 & 29.1 \\
$\mathbf{2 0 1 1}$ & 2682 & 26.5 \\
$\mathbf{2 0 1 2}$ & 2535 & 25.1 \\
$\mathbf{2 0 1 3}$ & 1954 & 19.3 \\
Total number & 10,110 & 100 \\
\hline
\end{tabular}

Table 2. Distribution of recorded suspects taken from Qena cities.

\begin{tabular}{ccc|}
\hline Place of residence & No. $(\mathbf{n}=\mathbf{1 0 , 1 1 0})$ & \% \\
\hline Abu-Tesht & 689 & 6.8 \\
Deshna & 800 & 7.9 \\
Farshoot & 779 & 7.7 \\
N Hamadi & 924 & 9.1 \\
Qena & 6387 & 63.2 \\
Qous & 531 & 5.3 \\
\hline
\end{tabular}

Table 3. Cause of referral for sputum direct smear examination.

\begin{tabular}{ccc}
\hline Reason for DSM & No. $(\mathbf{n}=\mathbf{1 0 , 1 1 0})$ & \% \\
\hline Contacts & 464 & 4.6 \\
cases & 9486 & 93.8 \\
Follow-up & 160 & 1.6 \\
\hline
\end{tabular}

Table 4. Total number of examined and excluded suspects.

\begin{tabular}{cc}
\hline & Suspects \\
\hline DSM1 (10110) & 10,110 \\
DSM2 (10110) & 10,110 \\
DSM3 (9420) & 9420 \\
Excluded & 690 \\
\hline
\end{tabular}

DSM1: direct smear $1^{\text {st }}$ sample, DSM2: direct smear $2^{\text {nd }}$ sample, DSM3: direct smear $3^{\text {rd }}$ sample. 


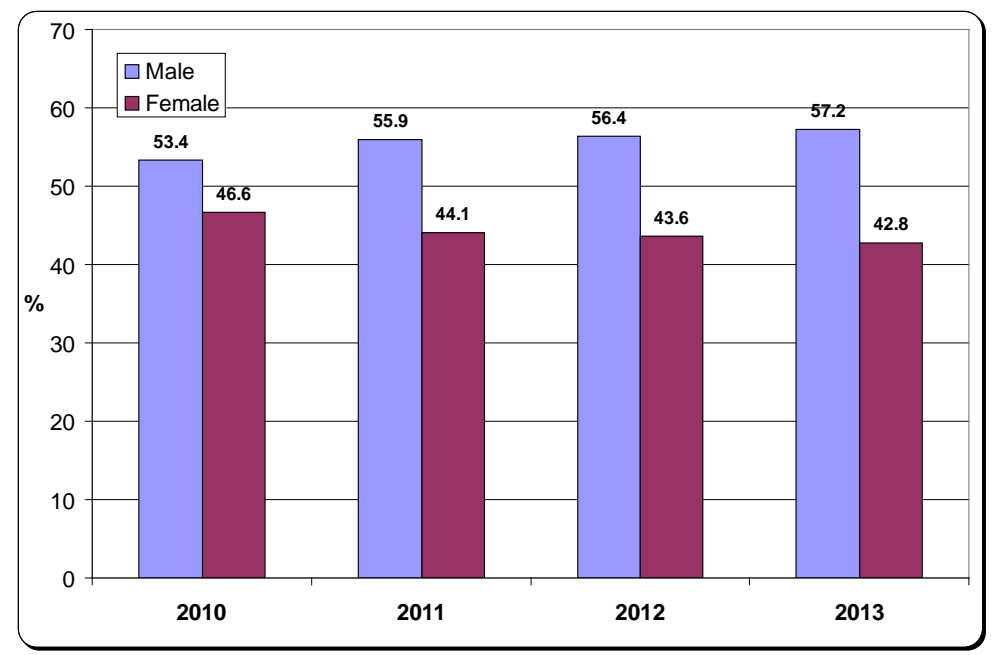

Figure 1. Gender distribution in the study years (2010-2013).

Table 5. Different combinations of the recorded direct smears (9420).

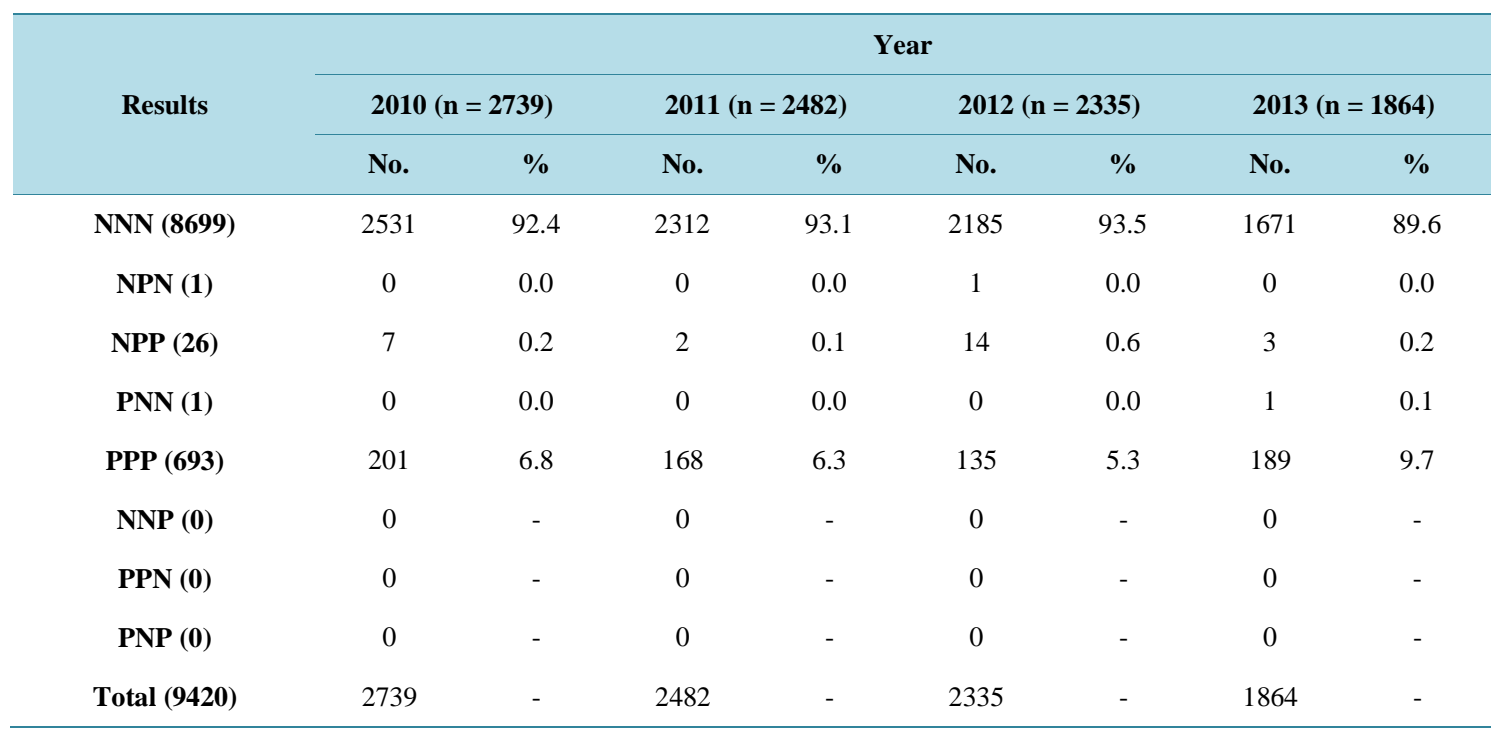

Table 6. Incremental yield of positive identified on first, second and third specimen.

\begin{tabular}{|c|c|c|c|c|c|c|c|c|}
\hline \multirow{3}{*}{ Results } & \multicolumn{8}{|c|}{ Year } \\
\hline & \multicolumn{2}{|c|}{$2010(n=2739)$} & \multicolumn{2}{|c|}{$2011(n=2482)$} & \multicolumn{2}{|c|}{$2012(n=2335)$} & \multicolumn{2}{|c|}{$2013(n=1964)$} \\
\hline & No. & $\%$ & No. & $\%$ & No. & $\%$ & No. & $\%$ \\
\hline Total number of three sputum smear $(9420)$ & 2739 & - & 2482 & - & 2335 & - & 1864 & - \\
\hline At least two positive (PPP, NPP) (719) & 208 & 7.6 & 170 & 6.8 & 149 & 6.4 & 192 & 10.3 \\
\hline At least one positive (PPP, NPP, NPN, PNN) (721) & 208 & 7.6 & 170 & 6.8 & 150 & 6.4 & 193 & 10.3 \\
\hline Positive in first smear (PPP, PNN) (694) & 201 & 7.3 & 168 & 6.8 & 135 & 5.8 & 190 & 10.2 \\
\hline Positive in second smear (NPP, NPN) (27) & 7 & 0.26 & 2 & 0.1 & 15 & 0.6 & 3 & 0.2 \\
\hline Positive in the third smear (NNP) (0) & 0 & 0 & 0 & 0 & 0 & 0 & 0 & 0 \\
\hline
\end{tabular}


For only 3.6\% of positive cases (26 of 719) a third specimen was required (NPP) to make a definitive diagnosis of TB, whereas in $96.4 \%$ (693 of 719) first sample was positive and a second confirmatory sample was required (Table 7).

\section{Discussion}

The present study is a four-year retrospective analysis of the results of sputum smear examination with an aim to determine the usefulness of examining the second and third DSM specimen. Guidelines of tuberculosis control recommend that microscopic examination of three sputum specimens is necessary for the evaluation of persons suspected of having pulmonary TB [16]. In developing countries where human and financial resources are significantly limited, considerable debate exists on reconsidering of examination of three sputum smears because more than one sputum sample was not rewarding [17] [18], resulting in extra burden and patient inconvenience. A study conducted in Zambia, found $77.1 \%$ AFB on first smear, a further $15 \%$ on the second smear and $7.9 \%$ additional cases were identified on the third smear [19]. A reanalysis of data from a study involving 42 laboratories in four high burden countries showed that incremental yield from a third smear ranged $0.7 \%-7.21 \%$ [20]. Nelson, found an incremental yield of around 5\% on third sputum examination [21]. A systematic review of 41 studies found that, on average, the second smear detected about $13 \%$ and the third smear detected $4 \%$ of all smear positive cases [22]. In our study 7.6\% (719 of 9420) of cases fulfilled the case definition, having at least one positive smear result confirmed by another positive smear examination. This was similar with the expected positivity in other studies [19] [23]. Of these, $96.4 \%$ were positive in the first smear, $3.6 \%$ in the second specimen which was confirmed by the third one. The need for confirmation of an AFB positive result by a second positive one to diagnose a case of TB has been challenged. Critics argued that this practice, result in fewer cases being put on treatment, considering the numbers that do not return after a first positive smear [24]. Three smears are helpful for case finding in those suspects where first or second sputum smear is negative [25]. Considering only first two smears, case detection rate came out to be $96.4 \%$ (693 of 9420) and a further 3.6\% (26 of 9420) fulfilled the case definition based on third smear in combination with second smear (NPP). A third sputum specimen is useful as confirmatory evidence if only one of the first two smears has a positive result. Therefore it appears that the WHO recommendation of collecting at least three sputum specimens from all patients with suspected PTB must be followed if only one of the first two smears has a positive result. Shivakumar et al. have concluded in their study that repeated follow-up smears are not essential since the incremental yield with a second spot sputum specimen is negligible, provided that the first smear is from early morning specimen [26]. It is likely that at our testing centre, approximately 6 positive cases per year would have been missed at one center alone if the second EM specimen was not examined (26 in 4 years, NPP). The detection of an additional smear positive patient annually by examining the second specimen is important since MDR-TB is a national emergency. Given that smear negative patients can also transmit tuberculosis though to a lesser degree, it is important to detect all smear positive patients [27]. Until such a time that rapid culture and drug susceptibility testing are routinely made available and affordable, the microscopic examination of a second early morning sputum specimen is essential even if the first was negative. Sputum smear negative examinations were uncommonly found to turn smear positive (NNP).

It is also important to discuss what happens to patients who have one positive slide out of three. Those with positive smear are infectious and are responsible for the spread of disease [25]. This makes the clinicians alert, these patients should have a further two specimens collected and if at least one is positive, the patient would be declared smear positive [20]. Isolated smear positive cases were rare in this study (2/9420). The possible reasons for these isolated smear positive cases could be that probability of finding acid-fast bacilli in sputum smears by microscopy is directly related to the concentration of bacilli in the sputum [29]. Type of specimen, its quality, quantity, bacterial content and viability of organism considerably influence the sensitivity and the specificity of the methods; and these in turn would vary under different diagnostic situations [30]. In this study no information

Table 7. Analysis of pulmonary TB with at least two positive smears (719).

\begin{tabular}{cccc} 
& Number & \% from total suspects (9420) & \% from positive cases (719) \\
\hline PPP & 693 & 96.4 & 7.4 \\
NPP & 26 & 3.6 & 0.2 \\
\hline
\end{tabular}


on sputum smear grading was reported. A poor quality spot specimen is more likely to be negative as compared to an early morning specimen from the same patient.

The implications of the study results on policy and practice are: The incremental yield of the second sputum smear was low whereas it was zero for the third sample. Given that Egypt is a resource-limited country and TB frequently occurs in socio-economically deprived populations, this will in fact benefit the patients by reducing the number of visits to health facilities as examination of other samples is usually done on additional visits. Hence, we will also benefit from the savings in cost and time incurred for the laboratory, which can be used for further improving quality of smear microscopy. In a study done in various microscopy centres of the state of Chattisgarh, India in 2012, decreasing the number of sputum examinations during follow-up can reduce the overall laboratory workload by $15 \%$ [31]. Therefore, we strongly recommend discontinuation of examining third sample if the first two were confirmed especially in areas where sputum culture could not be done.

The study had some limitations. There was no information on sputum smear grading which would have added additional value to our findings. No information on drug susceptibility profile was studied to find out the influence of drug resistance on the incremental yield of the second sputum smear examination. These results should be reassessed as part of a study involving different laboratories from all the regions in Egypt. The results of this study can be extrapolated only to areas that still use only sputum smear microscopy which is insensitive in comparison to sputum culture.

In conclusion, TB was diagnosed from the first sample with relatively higher frequency. The incremental yield of a second smear examination was low, while the third was negligible. We strongly recommend discontinuation of examining third sample if the first two were confirmed especially in areas where sputum culture could not be done. By reducing the number of smears needed, the costs could be reduced. Cost effectiveness is very important in developing countries like Egypt.

\section{References}

[1] Tenover, F.C., Crawford, J.T., Huebner, R.E., Geiter, L.J., Horsburgh Jr., C.R. and Good, R.C. (1993) The Resurgence of Tuberculosis: Is Your Laboratory Ready? Journal of Clinical Microbiology, 31, 767-770.

[2] Saceanu, C.A., Pfeiffer, N.C. and McLean, T. (1993) Evaluation of Sputum Smears Concentrated by Centrifugation for Detection of Acid-Fast Bacilli. Journal of Clinical Microbiology, 31, 2371-2374.

[3] Jost Jr., K.C., Dunbar, D.F., Barth, S.S., Headley, V.L. and Elliott, L.B. (1995) Identification of Mycobacterium tuberculosis and Mycobacterium avium Complex Directly from Smear-Positive Sputum Specimens and BACTEC 12B Cultures by High-Performance Liquid Chromatography with Fluorescence Detection and Computer-Driven Pattern Recognition Models. Journal of Clinical Microbiology, 33, 1270-1277.

[4] Reisner, B.S., Gatson, A.M. and Woods, G.L. (1994) Use of Gen-Probe Accu Probes to Identify Mycobacterium avium complex, Mycobacterium tuberculosis Complex, Mycobacterium kansasii, and Mycobacterium gordonae Directly from BACTEC TB Broth Cultures. Journal of Clinical Microbiology, 32, 2995-2998.

[5] Woods, G.L., Fish, G., Plaunt, M. and Murphy, T. (1997) Clinical Evaluation of Difco ESP Culture System II for Growth and Detection of Mycobacteria. Journal of Clinical Microbiology, 35, 121-124.

[6] Clarridge III, J.E., Shawar, R.M., Shinnick, T.M. and Plikaytis, B.B. (1993) Large-Scale Use of Polymerase Chain Reaction for Detection of Mycobacterium tuberculosis in a Routine Mycobacteriology Laboratory. Journal of Clinical Microbiology, 31, 2049-2056.

[7] Nolte, F.S. and Metchock, B. (1995) Mycobacteria. in Manual of Clinical Microbiology. In: Murray, P.R., Baron, E.J., Pfaller, M.A., Tenover, F.C. and Yolken, R.H., Eds., 6th Edition, American Society for Microbiology, Washington DC, 400-437.

[8] Kent, P.T. and Kubica, G.P. (1985) Public Health Mycobacteriology: A Guide for the Level III Laboratory. US Department of Health and Human Services, Centers for Disease Control, Atlanta.

[9] Shinnick, T.M. and Good, R.C. (1995) Diagnostic Mycobacteriology: Laboratory Practices. Clinical Infectious Diseases, 21, 291-299. http://dx.doi.org/10.1093/clinids/21.2.291

[10] Mathur, P., Sacks, L., Auten, G., Sall, R., Levy, C. and Gordin, F. (1994) Delayed Diagnosis of Pulmonary Tuberculosis in City Hospitals. Archives of Internal Medicine, 154, 306-310. http://dx.doi.org/10.1001/archinte.1994.00420030112011

[11] Ipuge, Y.A., Rieder, H.L. and Enarson, D.A. (1996) The Yield of Acid Bacilli from Serial Smears in Routine Microscopy Laboratories in Rural Tanzania. Transactions of the Royal Society of Tropical Medicine and Hygiene, 90, 258261. http://dx.doi.org/10.1016/S0035-9203(96)90239-4 
[12] Wu, Z.L. and Wang, A.Q. (2000) Diagnostic Yield of Repeated Smear Microscopy Examinations among Patients Suspected of Pulmonary TB in Shandong Province of China. The International Journal of Tuberculosis and Lung Disease, 4, 1086-1087.

[13] Dujardin, B., Haelterman, E., Van Damme, W., et al. (1997) The Adequacy of One Smear for Diagnosis of Pulmonary Tuberculosis. American Journal of Public Health, 87, 1234-1235. http://dx.doi.org/10.2105/AJPH.87.7.1234-a

[14] Central TB Division (2011) Managing the RNTCP in Your Area-A Training Course (Modules 1-4) and (Modules 1-9). Directorate General of Health Services, Ministry of Health and Family Welfare, Government of India.

[15] Aït-Khaled, N., Alarcón, E., Armengol, R., Bissell, K., Boillot, F., et al. (2010) Management of Tuberculosis. A Guide to the Essentials of Good Practice. Sixth Edition, International Union against Tuberculosis and Lung Disease, 6, 1-85.

[16] World Health Organization (2010) Treatment of Tuberculosis: Guidelines for National Programmes. 4th Edition, World Health Organization Document WHO/HTM/TB/2009 (420), 1-147.

[17] Crampin, A.C., Floyd, S. and Mwaungulu, F. (2001) Comparison of Two versus Three Smears in Identifying Culture-Positive Tuberculosis Patients in a Rural African Setting with High HIV Prevalence. The International Journal of Tuberculosis and Lung Disease, 5, 994-999.

[18] Harries, A.D., Mphasa, N.B. and Mundy, C. (2000) Screening Tuberculosis Suspects Using Two Sputum Smears. The International Journal of Tuberculosis and Lung Disease, 4, 36-40.

[19] Walker, D., McNerney, R. and Mwembo, M.K. (2000) An Incremental Cost-Effectiveness Analysis of the First, Second and Third Sputum Examination in the Diagnosis of Pulmonary Tuberculosis. International Journal of Tuberculosis and Lung Disease, 4, 246-251.

[20] Rieder, H.L., Chiang, C.Y. and Rusan, I.D. (2005) A Method to Determine the Utility of the Third Diagnostic and the Second Follow-Up Sputum Smear Examination to Diagnose Tuberculosis Cases and Failures. International Journal of Tuberculosis and Lung Disease, 9, 384-391.

[21] Nelson, S.N., Deike, M.A. and Cartwright, C.D. (1998) Value of Examining Multiple Sputum Specimens in the Diagnosis of Pulmonary Tuberculosis. Journal of Clinical Microbiology, 36, 467-469.

[22] Global Tuberculosis Control Report of Pakistan (2004).

[23] Van Cleeff, M.R.A., Kivihya Ndugga, L. and Githui, W. (2003) A Comprehensive Study of the Efficiency of the Routine Pulmonary Tuberculosis Diagnostic Process in Nairobi. The International Journal of Tuberculosis and Lung Disease, 7, 186-189.

[24] Squire, S.B., Kyasulu, I.K. and Kanyerer, H. (1996) Why Bother with Three Sputum Smears for Case Finding in TB Control. Transactions of the Royal Society of Tropical Medicine and Hygiene, 90, 478.

[25] Mabaera, B., Lauritsen, J.M. and Katamba, A. (2007) Sputum Smear-Positive Tuberculosis: Empiric Evidence Challenges the Need for Confirmatory Smears. The International Journal of Tuberculosis and Lung Disease, 11, 959-964.

[26] Shivakumar, M., Prabhakarareddy, B., Rajaprasannakumar, A., Vijayakumaran, P. and Krishnamurthy, P. (2006) Repeated Sputum Microscopy Is Not Essential for Monitoring Treatment Response. The International Journal of Tuberculosis and Lung Disease, 10, 1296-1298.

[27] Nataraj, G., Kanade, S., Parikh, R., Khatri, V., Mehta, P., et al. (2011) Incremental Yield in Sputum Smear Positivity by Examining a Second Early Morning Sputum Specimen in Follow-Up Patients on DOTS: 7-Year Analysis of RNTCP Laboratory Register. Indian Journal of Tuberculosis, 58, 60-65.

[28] Mabaera, B., Lauritsen, J.M. and Katamba, A. (2007) Sputum Smear-Positive Tuberculosis: Empiric Evidence Challenges the Need for Confirmatory Smears. The International Journal of Tuberculosis and Lung Disease, 11, 959-964.

[29] Deun, A.V., Zwahlen, M., Bola, V., et al. (2007) Validation of Candidate Smear Microscopy Quality Indicators, Extracted from Tuberculosis Laboratory Registers. The International Journal of Tuberculosis and Lung Disease, 11, $300-$ 305.

[30] Deun, A.V., Salim, A.H., Cooreman, E., et al. (2004) Scanty AFB Smears: What's in a Name? The International Journal of Tuberculosis and Lung Disease, 8, 816-823.

[31] Kundu, D., Kumar, A.M.V., Satyanarayana, S., Dewan, P.K., Nair, S.A., Khaparde, K., Nayak, P., Van den Bergh, R., Manzi, M., Enarson, D.A., Rao Deshpande, M. and Chandraker, S. (2012) Can Follow-Up Examination of Tuberculosis Patients Be Simplified? A Study in Chhattisgarh, India. PLOS ONE, 7, e51038. http://dx.doi.org/10.1371/journal.pone.0051038 